\title{
Recognition of Fault Signature Patterns Using Fuzzy Logic for Prevention of Breakdowns in Steel Continuous Casting Process
}

\author{
Arya K. Bhattacharya, P.S. Srinivas, K. Chithra, S.V. Jatla, and Jadav Das \\ Automation Division, Tata Steel, Jamshedpur 831001, India \\ \{arya, srini, chithra, shiva, jadav\}@aut.tatasteel.com
}

\begin{abstract}
In the continuous casting process of steel, a thin solid shell gradually forms around the cooling copper mould inner perimeter as liquid steel flows through it. By the time steel emerges from mould bottom, the perimetric solid shell acquires sufficient thickness and strength to support ferrostatic pressure of liquid steel in its interior. However, the formative shell can tear, stick to mold wall or fail to thicken adequately - in all cases leading to massive leakage of liquid steel out of mold bottom and consequent breakdown of continuous casting. All such phenomena leading to process disruption are preceded by typical patterns in temperature history data recorded at arrays of thermocouples embedded in mould walls. A fuzzy system is developed to accurately recognize these patterns in real time and regulate the speed of casting to prevent breakdowns. The system designed and implemented is fail-safe, has a low false-alarm ratio, and provides online diagnostics of the continuous casting process.
\end{abstract}

\section{Introduction}

Continuous casting in the steel industry is the process whereby molten steel is solidified into 'semifinished' slabs for subsequent rolling in finishing mills downstream.

Molten steel is poured into a mold of rectangular cross-section and length of 900 $\mathrm{mm}$. Water flowing in embedded pipes in the mold transfers heat out from the solidifying steel through a copper lining on mold inner wall. Steel that is close to the mold walls gets solidified and forms a shell around the liquid interior, thus forming a downwards-moving semi-solidified steel slab called the strand. The main function of the mold is to establish a solid shell sufficient in strength to contain the liquid core when the strand exits from the mold into secondary cooling zones downstream.

When the partially solidified strand comes out of the mold the solid shell has to withstand the ferrostatic pressure of liquid steel within the strand above. If this shell cannot withstand the pressure due to any abnormality in solidification, it may cause a leak leading to a 'breakout' - a spillage of liquid steel from and around mold bottom. Alternatively, in the early stages of solidification in the mold the formative shell may tear leading to liquid steel leaking onto the mold wall and consequent breakouts. Breakouts of the first type are referred to as crack or thin-shell breakouts, while those of the second type are called sticker breakouts. Either way, a breakout is a catastrophic incident and needs to be avoided. Consequently, all modern continuous casting systems are equipped with on-line breakout detection systems - BDS. 
A BDS [1-3] receives the temperature profile of the copper mold walls usually at two or three horizontal levels through a series of thermocouples that encompass the mold perimeter at that level. The temperature readings are obtained at very small time intervals. The BDS analyzes in real time the temperature time-history and concludes whether a breakout is likely or not. If likely, it commands a reduction of the casting speed to near-zero to heal the sticker/shell-weakness and prevent the breakout.

Breakouts are a consequence of a complex combination of metallurgical, chemical and mechanical phenomena and operational characteristics. These vary continuously with time as casting proceeds. The temperature time series matrix, that may be formed by representing in each row a thermocouple's temperature history - reflects in some sense, i.e. is a function of, the internal dynamics of these nonlinear physical phenomena. Almost all existing BD systems map this matrix in real time into a binary output alarming or no alarming - using some form of pattern recognition.

The Breakout Detection systems existing worldwide vary from one another primarily in the nature of the above mapping. A paradigm of these binary-output mappings is the neglect of continuity, i.e. the fact that the continuous evolution of the physical phenomena - and consequent temperature time matrix - naturally transforms into a continually evolving 'tendency to breakout'. In this development, fuzzy logic is used to transform the features of this matrix into a value of 'breakoutability', an index on 0-100 scale of this continuous 'tendency to breakout'. When this value crosses a threshold, an alarm is raised.

Expert knowledge of the relationship between casting physics and casting health is 'banded' rather than 'precise', i.e. if input variable $i$ is within bounds $\left[\mathrm{L}_{\mathrm{i}}, \mathrm{U}_{\mathrm{i}}\right]$, for all $i$ in the input variable space, then the breakoutability can be said to lie within bounds $\left[\mathrm{L}_{\mathrm{o}}, \mathrm{U}_{\mathrm{o}}\right]$. A binary system imposes undue determinism that can be misplaced leading either to detection failure or excessive false alarming. Fuzzy logic is the correct conceptual formalism for representing this form of knowledge. It captures both the natural continuity of fault evolution and the form of expert knowledge of the process, in effect transforming a fault detection system into a real time casting health diagnostics system.

The next section describes the methodology of the developed breakout detection system, named as Casting Online Diagnostics System or CODS. The results of testing are described in section three. The last section concludes the discussion.

\section{Casting Online Diagnostics System}

The conceptual architecture of the developed CODS is shown in Fig. 1. Thermocouples embedded in mold walls at two horizontal layers provide temperature inputs in real time. They serve as common inputs to three fuzzy modules for detection of the three types of breakouts - sticker, crack and thin-shell, and to an intelligent agent that is also based on fuzzy logic. The three modules and the intelligent agent are described below. Apart from temperatures, the three modules also take parameters like casting speed and percentage carbon as inputs. Each of these modules generates a value of instantaneous maximum breakoutability, of type corresponding to the module, as output. The outputs from each module are composed in the breakoutability analyzer, 


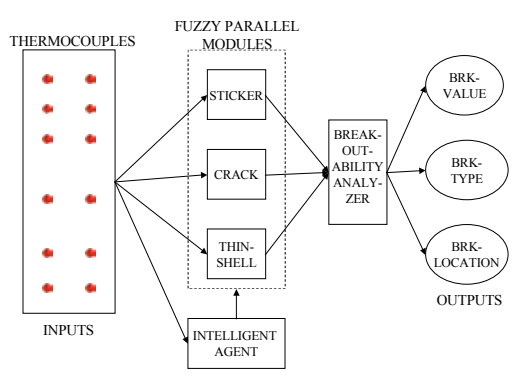

Fig. 1. The CODS Architecture

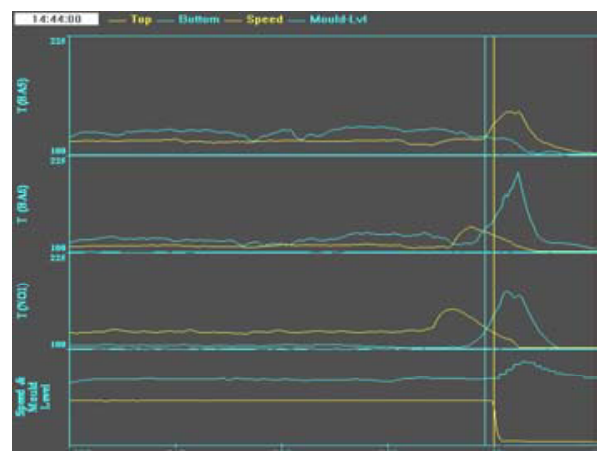

Fig. 2. Temperature time-history pattern of a Sticker

which generates in real-time a value of overall maximum breakoutability, its type and location of the point of maximum abnormality in the mold. These results are displayed to the operator on a HMI screen.

The description of each of the three modules is provided below.

\section{Sticker Module}

A sticker is accompanied by a specific pattern in the temperature time series of the thermocouple it closely passes by. This is illustrated in the snapshot in Fig. 2. The top three of four plots in this figure show temperatures (y-axis) against time (in secs). Since a sticker corresponds to a tear in the formative shell that exposes liquid steel at a higher-than-usual temperature to the mold wall, it shows up as a high-temperature pulse peaking at the instant the sticker is closest to the thermocouple during its downward trajectory. The temperature gradient shoots up appreciably as the sticker approaches, and then falls off. These are shown in the blue lines in the second and third graphs - each representing temperatures from a thermocouple in the second horizontal layer. The yellow lines (darker in black-and-white print) represent temperatures from the first layer of thermocouples.

The sticker fuzzy module compares instantaneous temperature-time patterns against this typical sticker signature. In a computation cycle, this module runs over all thermocouples. In each thermocouple, it takes three inputs, first, the extent of temperature rise, second, the temperature gradient, and third, the percentage of Carbon in the steel. (The properties of steel vary with $\% \mathrm{C}$ ). The breakoutability, i.e. the output, varies directly with all three inputs and is related to them through a fuzzy associative rule matrix. In a sense, the breakoutability represents the 'degree-of-conformity' of an instantaneous temperature-time pattern with the defined sticker signature. A breakoutability value greater than 85 in the second layer generates an alarm.

The variables temperature rise and gradient are both normalized into the [-1:1] space by comparison against their respective bounds of existence. The normalized space for both inputs is discretized using 7 triangular sets as shown in Fig. 3. The variable '\%Carbon' is divided into 3 fuzzy sets in a manner consistent with the variation of steel properties with Carbon. 


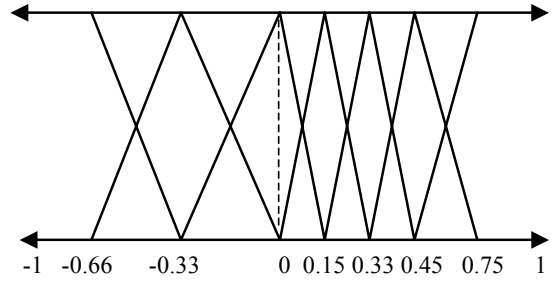

Fig. 3. Fuzzy membership functions for first and second inputs, namely temperature rise and rate of rise, to sticker module

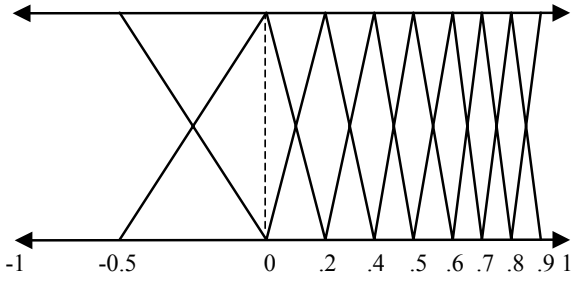

Fig. 4. Fuzzy membership functions for output breakoutability, common to all breakout prediction modules

The output variable 'breakoutability' is scanned by 9 fuzzy membership functions (MF) traversing its domain of existence from 0 to 100 . Since 85 to 90 is the alarming threshold (depending on the module), the MF are concentrated in the region above 75 , while only 2 MF scan the space below 50. This distribution is shown in Fig. 4.

The fuzzy rule matrix for sticker is shown in Table 1 . The columns represent MF of the first input, i.e. temperature rise, while the rows represent rate of rise. The third input, Carbon, is incorporated in each element of the table. It may be seen that each element is composed of three integers. The first denotes the activated MF of the output variable when Carbon lies in the first MF. The second and third integers denote the value added to the first integer to obtain the output MF when Carbon belongs to the second or third MF.

\section{Crack Module}

A typical crack signature is illustrated in Fig. 5. A crack (or any depression) is associated with an insulation in heat flux between strand shell and mold, resulting in low heat transfer from that region and consequently a thinner and weaker shell. The recorded temperatures show a dip followed by a rise as the crack passes by the

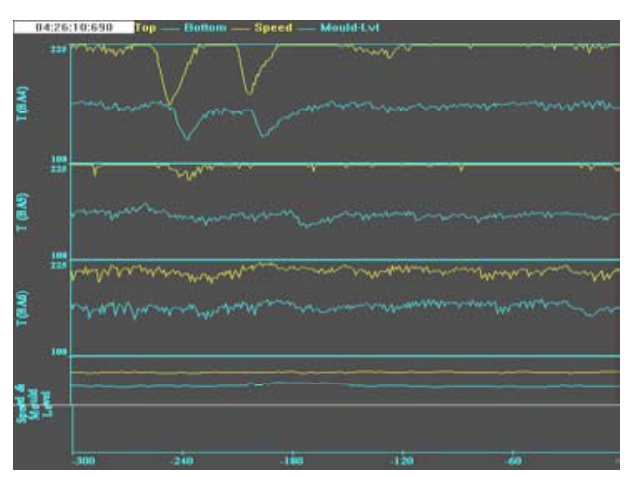

Fig. 5. Temperature time-history pattern for a Crack
Table 1. Fuzzy Associative Matrix for sticker module

\begin{tabular}{|l|l|l|l|l|l|l|}
\hline $1^{0,1}$ & $2^{0,1}$ & $3^{1,2}$ & $5^{1,3}$ & $6^{1,3}$ & $8^{0,1}$ & 9 \\
\hline $1^{0,1}$ & $1^{0,1}$ & $2^{1,2}$ & $3^{1,2}$ & $4^{1,3}$ & $6^{1,3}$ & $8^{0,1}$ \\
\hline $1^{0,1}$ & $1^{0,1}$ & $1^{0,1}$ & $2^{1,2}$ & $2^{1,3}$ & $4^{1,3}$ & $6^{1,3}$ \\
\hline $1^{0,1}$ & $1^{0,1}$ & $1^{0,1}$ & $1^{0,1}$ & $2^{1,2}$ & $3^{1,2}$ & $5^{1,3}$ \\
\hline 1 & 1 & 1 & $1^{0,1}$ & $1^{0,1}$ & $1^{0,1}$ & $3^{0,1}$ \\
\hline 1 & 1 & 1 & 1 & $1^{0,1}$ & $1^{0,1}$ & $2^{0,1}$ \\
\hline 1 & 1 & 1 & 1 & 1 & 1 & $1^{0,1}$ \\
\hline \multicolumn{7}{|c}{ Increasing Int One $\longrightarrow$} \\
\hline \multicolumn{7}{|c}{}
\end{tabular}


thermocouple. This is shown in the first graph in Fig. 5 - which shows two cracks passing in quick succession.

The fuzzy module for cracks is designed to recognize the typical crack signature. It takes four inputs. First, the extent of temperature dip, second, the casting speed, and third, \%C. The last input is the layer location relative to total mold length, a fraction varying from 0 to $1 ; 0$ corresponds to mold top. The output varies directly with all four inputs, and a threshold value of 90 is considered for alarming.

\section{Thin Shell Module}

A thin shell cannot be easily distinguished as a pattern on a thermocouple. Here the shell across its perimeter, at a given layer, is thinner than normal under same casting condi-tions, and if this thinness falls below a threshold, the layer will be unable to support the ferro-static pressure of the molten core above. A thin shell implies that the molten core is closer to mold wall during casting than is normal, and hence the instantaneous average layer temperature taken over all thermocouples in the layer will be higher than the 'normal average'. In other words, the time-localized average layer temperature varies inversely as average layer physical thickness, and the deviation of this temperature from a 'normal average' is a mapping of the degree of thinness or thickness of the shell. Thus if a method can be found to evaluate this 'normal average' temperature, a measure of shell thinness can be obtained.

The normal average temperature is a strong function of casting parameters after filtering out transients. It varies dynamically as casting parameters change. An intelligent agent (see Fig. 1) is used to evaluate it. This agent is essentially a Takagi-Sugeno type fuzzy system and is imbued with the ability to quickly learn the caster features and dynamically adapt to casting conditions while neglecting transients.

In the fuzzy module for thin-shell breakoutability evaluation, the first input is the difference between instantaneous and normal average temperatures. The second, third and fourth inputs are, respectively, the casting speed, \%Carbon, and vertical position. The output is breakoutability, varying directly with all inputs.

At every computation cycle, the three fuzzy modules generate in parallel a value of breakoutability corresponding to each type, along with the location of the point (thermocouple) of maximum breakoutability. These are sent to the breakoutability analyzer (Fig. 1), which decides on the global maximum as well as its point of location in the mold. These are displayed to the operator through a HMI.

The global maximum breakoutability, along with its type and location, serve as inputs to a speed control module which commands changes in casting speed, at deceleration rates based on values of these parameters. .

\section{Results and Validation}

The CODS has been implemented in the slab casters of Tata Steel after passing through a rigorous validation process. An earlier version of a BDS [5] was installed in the premises. This showed good performance in detecting stickers, but did not detect other types of breakouts. Further, the number of false alarms was an area of concern. 
The earlier BDS was used as a baseline for validation of the new system. For a period of two months, thermocouple data along with other casting parameters was sent to both detection systems installed on parallel computing platforms, and outputs from both were analyzed. Only outputs from the previous system were sent to speed control units. When either system raised an alarm, the corresponding slab that was cast at the time of alarm was visually surveyed for tell-tale marks of stickers or cracks. This was to confirm whether the alarm was true or false. In some cases both systems raised alarms at near-identical instances, in other cases alarms raised by one system found no mention in the other.

To rationally analyze the consequent data, a few conceptual cases were constructed and every alarm was classified into one or other of these cases [6]. A qualitative value on the validation scale - for the new BDS - was attributed to each of these cases, and at the end of the testing period the results were summarized to decide if the new BDS had passed the acceptance criteria. Table 2 lists out the cases. The second column in this table provides the feature - or definition - of the case. The last column states its implication towards system acceptance. In the table, ES and NS denote Earlier and New Systems, respectively.

Table 2. Validation Strategy: Definition of cases and impact on acceptance

\begin{tabular}{|c|c|c|}
\hline Cases & Definition & $\begin{array}{c}\text { Implication on Ac- } \\
\text { ceptance }\end{array}$ \\
\hline A & ES $^{1}$ : False Alarm & None \\
\hline B & ES: True Alarm & Indirect \\
\hline C & NS: Missed True Alarm & Unacceptable \\
\hline D & NS: True Alarm & Desirable \\
\hline E & $\begin{array}{c}\text { Breakout: ES Missed, } \\
\text { NS Captured }\end{array}$ & $\begin{array}{c}\text { Desirable from va- } \\
\text { lidation viewpoint }\end{array}$ \\
\hline F1 & NS: False Alarm & Less the better \\
\hline F2 & NS: Desirable ${ }^{2}$ Alarm & Desirable \\
\hline G & $\begin{array}{c}\text { Breakout: ES Missed, } \\
\text { NS also Missed }\end{array}$ & Unacceptable \\
\hline
\end{tabular}

Table 3. Testing Results Summary: Sixty days continuous casting

\begin{tabular}{|c|c|c|c|c|c|}
\hline \multirow{2}{*}{$\begin{array}{c}\text { Detec- } \\
\text { Slabs in- } \\
\text { tion } \\
\text { Spected } \\
\text { System }\end{array}$} & \multicolumn{2}{|c|}{ Stickers } & \multicolumn{2}{|c|}{$\begin{array}{c}\text { Cracks and } \\
\text { alarm }\end{array}$} & Thin Shells \\
\cline { 3 - 6 } & True & False & True & False \\
\hline $\begin{array}{c}\text { Earlier } \\
\text { BDS }\end{array}$ & 36 & 7 & 29 & NA & NA \\
\hline $\begin{array}{c}\text { New } \\
\text { BDS: } \\
\text { CODS }\end{array}$ & 24 & 7 & 5 & 8 & 4 \\
\hline
\end{tabular}

The defined cases cover the entire space of conditional possibilities. It is seen that Cases $\mathrm{C}$ and $\mathrm{G}$ are roadblocks to acceptance. When a true alarm is raised in both systems, Cases B and D occur simultaneously. The converse is also expected to occur sometimes, i.e. a false alarm is raised in both systems leading to Cases A and F1 being flagged at the same time.

The results of testing are summarized in Table 3. The second column presents total number of alarms generated by either system. It may be noted that some of these alarms were common to the two systems, as discussed above. The next two columns show sticker alarms from either system, and classify them into true or false based on inspection. The last two columns show crack and thin shell alarms from only the new system, as these are not captured by the earlier one.

In the concerned period of testing there was no occurrence of breakout. The seven true sticker alarms were common to both systems, and hence belong to Cases B and D. There was no occurrence of Case C (Table 2). The number of false sticker alarms 
has come down. There were eight cases of desirable crack alarms, and four cases of undesirable ones. Most importantly, there were no occurrences of Cases $\mathrm{C}$ and $\mathrm{G}$ - the unacceptable conditions; and the number of false alarms are seen to have reduced significantly. In other words, the new BDS passed the acceptance criteria for implementation.

\section{Conclusions}

Application of the concepts and principles of fuzzy logic has facilitated the development and implementation of an advanced breakout detection system for continuous casting of steel. Signatures on temperature time-history data specific to each type of breakout are compared in real time against instantaneous patterns on each thermocouple, to provide a 'degree of conformity'. When the conformity exceeds a threshold, an alarm is generated.

This breakout detection system successfully prevents all types of breakouts, and hence satisfies the fundamental requirement of a reliable breakout detection system. It also raises low number of false alarms in comparison to existing BD systems.

Furthermore, the developed system provides operational personnel with real-time diagnostic information on health of casting and evolution of potential faults. Accordingly, it is named as Casting Online Diagnostics System, or CODS.

\section{Acknowledgment}

The authors acknowledge their gratitude to Tata Steel for continuous support and encouragement in this work.

\section{References}

1. J.D.Madill, "Application of Mold Thermal Monitoring to Avesta Sheffield SMACC Slab Caster", 1996 Vol. 23, No. 3, Ironmaking and Steelmaking, pp. 223-234.

2. M.J.Lu, K.J.Lin., "Sticker Breakout Theory and its Prediction in Slab Continuous Casting", 1993 Steelmaking Conference Proceedings, pp. 343-352.

3. Richard T. Grass, George W. Skoczylas, "Reduction of Breakouts at Ispat Inland Inc", 2001 Steelmaking Conference Proceedings, pp. 41-52.

4. P.S. Srinivas, K. Chithra, Shiva Jatla, A.K. Bhattacharya, "Global Design for mould breakout detection system for slab casters", Tata Steel Internal Report No. AUT/PRJ/01019/GDD, July 2004.

5. Mahashabde, V.V., Bhaduri, R., Das, S., Kukreja, B.K., Agarwal, A.K., "Breakout Detection System at Tata Steel's slab caster using Neural Network", Tata Search, 1999, pp. 91-94.

6. P.S. Srinivas, K. Chithra, Shiva Jatla, A.K. Bhattacharya, "Field Testing Strategy for Mould Breakout Detection System", Tata Steel Internal Report No. AUT/PRJ/01019/FTS, Sep 2003. 